OPEN ACCESS

Edited by:

Graziano Pesole,

University of Bari Aldo Moro, Italy

Reviewed by:

Alexander Grigoryevich

Tonevitsky,

National Research University Higher

School of Economics, Russia

Hsiuying Wang,

National Chiao Tung University,

Taiwan

*Correspondence:

Aniello Russo

aniello.russo@unicampania.it

Nicoletta Potenza

nicoletta.potenza@unicampania.it

Specialty section:

This article was submitted to

$R N A$,

a section of the journal

Frontiers in Genetics

Received: 10 March 2021

Accepted: 19 April 2021

Published: 07 June 2021

Citation:

Siniscalchi C, Di Palo A, Russo A and Potenza N (2021) Human

MicroRNAs Interacting With

SARS-CoV-2 RNA Sequences:

Computational Analysis

and Experimental Target Validation.

Front. Genet. 12:678994.

doi: 10.3389/fgene.2021.678994

\section{Human MicroRNAs Interacting With SARS-CoV-2 RNA Sequences: Computational Analysis and Experimental Target Validation}

\author{
Chiara Siniscalchi, Armando Di Palo, Aniello Russo* and Nicoletta Potenza* \\ Department of Environmental, Biological and Pharmaceutical Sciences and Technologies, University of Campania "Luigi \\ Vanvitelli", Caserta, Italy
}

Severe acute respiratory syndrome coronavirus 2 (SARS-CoV-2) is a novel RNA virus affecting humans, causing a form of acute pulmonary respiratory disorder named COVID-19, declared a pandemic by the World Health Organization. MicroRNAs (miRNA) play an emerging and important role in the interplay between viruses and host cells. Although the impact of host miRNAs on SARS-CoV-2 infection has been predicted, experimental data are still missing. This study started by a bioinformatics prediction of cellular miRNAs potentially targeting viral RNAs; then, a number of criteria also based on experimental evidence and virus biology were applied, giving rise to eight promising binding miRNAs. Their interaction with viral sequences was experimentally validated by transfecting luciferase-based reporter plasmids carrying viral target sequences or their inverted sequences into the lung A549 cell line. Transfection of the reporter plasmids resulted in a reduction of luciferase activity for five out of the eight potential binding sites, suggesting responsiveness to endogenously expressed miRNAs. Co-transfection of the reporter plasmids along with miRNA mimics led to a further and strong reduction of luciferase activity, validating the interaction between miR-219a-2-3p, miR-30c-5p, miR-378d, miR-29a-3p, miR-15b-5p, and viral sequences. miR-15b was also able to repress plasmid-driven Spike expression. Intriguingly, the viral target sequences are fully conserved in more recent variants such as United Kingdom variant B.1.1.7 and South Africa 501Y.V2. Overall, this study provides a first experimental evidence of the interaction between specific cellular miRNAs and SARS-CoV-2 sequences, thus contributing to understanding the molecular mechanisms underlying virus infection and pathogenesis to envisage innovative therapeutic interventions and diagnostic tools.

Keywords: RNA virus, microRNA, COVID-19, SARS-CoV-2, anti-viral, non-coding RNA

\section{INTRODUCTION}

Severe acute respiratory syndrome coronavirus 2 (SARS-CoV-2) is an emerging RNA virus belonging to the Betacoronavirus family, first discovered in Wuhan, China, in December 2019 (Wu et al., 2020; Zhu et al., 2020). It is an exceptionally infectious virus that has rapidly spread across the world causing a form of acute pulmonary respiratory disorder named COVID-19 
(coronavirus disease 2019) that was declared a pandemic by the World Health Organization (WHO) on March 11, 2020. SARS-CoV-2 infection leads to a wide variety of clinical syndromes primarily involving the respiratory tract, ranging from asymptomatic infection to acute respiratory distress syndrome and death (approximately 3.7\% mortality rate, WHO 2020). Since the declaration of the pandemic, which severely affected people health worldwide and caused global economic recession, there has been an enormous effort to investigate the virus biology in order to develop treatments and vaccines.

COVID-19 is transmitted when droplets from an infected person penetrate the respiratory tract or mucous membrane of the eyes, nose, or mouth of another person. The preferential tropism of the virus for the airways and lung epithelial cells resides in the mechanism of virus entry into the host cell: the virus uses its viral spike glycoprotein (S protein) to bind to hostcell angiotensin-converting enzyme 2 (ACE2), after which host type II transmembrane serine protease TMPRSS2 cleaves the $\mathrm{S}$ protein to facilitate membrane fusion; co-expression of both ACE2 and TMPRSS2 occurs in only a minority of cells, primarily secretory epithelial cells in the upper and middle respiratory tract (Hoffmann et al., 2020; Sungnak et al., 2020; Ziegler et al., 2020).

Inside the host cell, it is well recognized that viruses employ a large array of cellular components to assist genome replication, protein synthesis, and viral particle assembly. These include endoplasmic reticulum, ribosomes, tRNAs, and other cytosolic factors but also regulatory molecules such as transcription factors and microRNAs (miRNA). Canonical gene expression regulation by miRNAs is based on their binding to complementary sequences on RNA targets, requiring a perfect match with the $2-8$ nucleotides of the miRNA $5^{\prime}$ region (seed region); then, miRNAs mediate translational repression and/or degradation of the RNA targets (Rzeszutek and Singh, 2020). As posttranscriptional regulators, miRNAs play an important role in a broad array of cellular functions, including the complex molecular interplay that is established upon viral infection of host cells (Umbach and Cullen, 2009; Trobaugh and Klimstra, 2017). Cellular miRNAs acting on viral RNAs have in fact been identified for human immunodeficiency virus (HIV) (Ahluwalia et al., 2008; Nathans et al., 2009), hepatitis C virus (Murakami et al., 2009; Li et al., 2016), hepatitis B virus (HBV) (Zhang et al., 2010; Potenza et al., 2011), papillomavirus (Nuovo et al., 2010), and many other viruses (Russo and Potenza, 2011; Trobaugh and Klimstra, 2017), including RNA viruses causing respiratory pathology such as influenza virus $\mathrm{H} 1 \mathrm{~N} 1$ and Rhino virus (Bhattacharyya and Biswas, 2020). In the case of HIV and HBV, there is convincing evidence that the identified miRNAs have been co-opted by the virus to fine-tune its replication, keeping it low to escape the immune system and establish a persistent infection (Huang et al., 2007; Mosca et al., 2014; Sagnelli et al., 2018).

To date, no experimental data have been published about microRNAs targeting SARS-CoV-2 RNA genome. However, some reports have predicted the potential of miRNA studies in the virus infection (Arisan et al., 2020; Chow and Salmena., 2020; Demirci and Adan, 2020; Guterres et al., 2020; Hosseini Rad Sm and McLellan, 2020; Jafarinejad-Farsangi et al., 2020;
Nersisyan et al., 2020; Pierce et al., 2020). Among the computational analyses, the study of Chow and Salmena (2020) reported a list of 128 miRNAs potentially targeting the SARS-CoV-2 genome resulting by analysis with computer programs TargetScan and RNA22 (Chow and Salmena, 2020); another study used programs miRDB and RNAhybrid to identify 288 miRNAs with putative binding sites within the viral genome (Pierce et al., 2020). The miRanda program was used in another work and predicted 160 miRNAs targeting the viral genomic RNA (Jafarinejad-Farsangi et al., 2020). These studies encourage further investigation, but the extremely high number of predicted miRNAs makes difficult their experimental validation. We then undertook a new bioinformatic search with more restrictive parameters and taking into account the virus biology with the purpose of identifying a limited number of promising miRNAs to be immediately validated by experimental procedures.

\section{MATERIALS AND METHODS}

\section{Computational Analyses}

Prediction of microRNA target sites was performed with RNAhybrid 2.2 (Rehmsmeier et al., 2004; Krüger and Rehmsmeier, 2006), using a cutoff parameter for free energy of binding $\mathrm{mfe}<-25 \mathrm{kcal} / \mathrm{mol}$, and MirTarget (Ivashchenko et al., 2014). The latter was used remotely within the miRDB database ${ }^{1}$, an online database for miRNA target prediction and functional annotations (Chen and Wang, 2020), using the predefined threshold score of 50. Conservation of the miRNA targets within the SARS-CoV-2 population was estimated by Blast searches of nucleotide collections at NCBI using as queries the entire miRNA-pairing sequences reported in Figure 1. Search was restricted to SARS-CoV-2, whose number of deposited sequences was 380 at the time of the analysis, by using its taxonomy ID (2697049). Blast-identified sequences were then downloaded and examined to verify target conservation. Nucleotide sequences of severe acute respiratory syndrome coronavirus (SARS-CoV) and Middle East respiratory-related coronavirus (MERS-CoV) genomes were downloaded from NCBI with accession numbers NC_004718.3 and NC_019843.3, respectively. Lung microRNA expression data were extracted from the Human miRNA Tissue Atlas (Ludwig et al., 2016). This is a web-based repository ${ }^{2}$ of experimental data collected by microarray analysis for detection of 1997 microRNAs in 61 tissues biopsies of different organs collected postmortem from two individuals.

\section{Reporter Constructs}

The viral segments supposed to be targeted by human miRNAs were obtained by chemical synthesis of complementary oligonucleotides (Invitrogen, Thermo Fisher Scientific) containing upstream XhoI and EcoRV restriction sites and a downstream NotI site (Potenza et al., 2016). Each couple of oligonucleotides, representing the target sites for the different miRNAs reported in Figure 1, was annealed and ligated into

\footnotetext{
${ }^{1}$ http://mirdb.org

${ }^{2}$ https://ccb-web.cs.uni-saarland.de/tissueatlas/
} 


\begin{tabular}{|c|c|c|c|c|c|c|}
\hline Human miRNA & $\begin{array}{l}\text { SARS-CoV-2 } \\
\text { genomic } \\
\text { target (nt) }\end{array}$ & $\begin{array}{l}\text { Target } \\
\text { ORF }\end{array}$ & $\begin{array}{l}\text { Mfe } \\
\text { (kcal/ } \\
\text { mol) }\end{array}$ & $\begin{array}{l}\text { MirTarget } \\
\text { score }\end{array}$ & $\begin{array}{c}\text { Lung } \\
\text { expression }\end{array}$ & miRNA-mRNA pairing \\
\hline miR-219a-2-3p & $1367-1393$ & $1 a$ & $-28,4$ & 76 & 6 & $\begin{array}{ccc}\text { mRNA: } & \text { 5'AUUUAUUGUCCAGCAUGUCACAAUUCA3' } \\
& : \quad: \quad::::::::: \quad:::::::: \\
\text { miRNA }: & \text { 3'UGUCU-ACAGGUCG----GUGUUAAGA5' }\end{array}$ \\
\hline $\operatorname{miR}-30 c-5 p$ & $4948-4981$ & $1 a$ & $-25,5$ & 95 & 158 & $\begin{array}{ccc}\text { mRNA: } & \text { 5'UUUGAGAGAAGUG-AGGACUAUUAAGGUGUUUACA3' } \\
& ::::::::::::::::: \\
\text { miRNA }: & 3^{\prime} \text { CGACUCU---CACAUCCU--------ACAAAUGU5' }\end{array}$ \\
\hline $\operatorname{miR}-122-5 p$ & $5294-5316$ & $1 \mathrm{a}$ & $-30,9$ & 67 & 177 & $\begin{array}{cc}\text { mRNA: } & \text { 5'GCCACUGCAUUGUUAACACUCCA3' } \\
& :: \quad:::::::::::::: \\
\text { miRNA }: & \text { 3'GUUUGUGGUAACAG-UGUGAGGU5' }\end{array}$ \\
\hline miR-378d & $11024-11054$ & 1a & $-28,4$ & 55 & 42 & 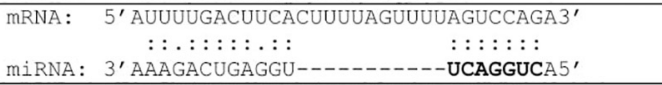 \\
\hline $\operatorname{miR}-29 a-3 p$ & 19459-19485 & $1 \mathrm{~b}$ & $-28,1$ & 91 & 3329 & 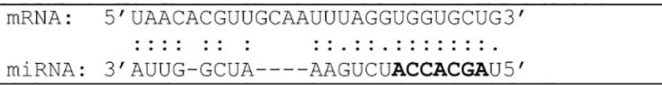 \\
\hline $\operatorname{miR}-181 d-5 p$ & $20766-20790$ & $1 \mathrm{~b}$ & $-27,9$ & 72 & 37 & $\begin{array}{cc}\text { mRNA: } & \text { 5'ACCUAAAGGCAUAAUGAUGAAUGUC3' }^{\prime} \\
& :::: \quad: \quad:: \quad: \ldots::::::: \\
\text { miRNA }: & 3^{\prime} \text { UGGGUGGCUGU--UGUUACUUACAA5' }\end{array}$ \\
\hline $\operatorname{miR}-15 b-5 p$ & $24074-24101$ & $S$ & $-28,9$ & 99 & 361 & 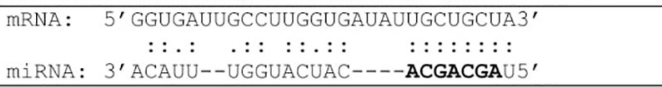 \\
\hline miR-103a-3p & $28705-28741$ & 10 & $-29,1$ & 85 & 149 & $\begin{array}{ccc}\text { mRNA: } & \text { 5' UCACAUUGGCACCCGCAAUCCUGCUAACAAUGCUGCA3' } \\
& :::::::: \quad::::::::::: \\
\text { miRNA: } & \text { 3'AGU---AUCG---------GGACA--UGUUACGACGA5' }\end{array}$ \\
\hline
\end{tabular}

FIGURE 1 | Prediction of human microRNA targets in the genomic RNA of SARS-CoV-2. Selection was performed with RNAhybrid 2.2 (Krüger and Rehmsmeier, 2006) and MirTarget (Chen and Wang, 2020). Seed regions are marked in bold. Nucleotide numbering refers to isolate Wuhan-Hu-1, complete genome (NC_045512.2). ${ }^{1}$ Microarrays data from the Human miRNA Tissue Atlas at https://ccb-web.cs.uni-saarland.de/tissueatlas/ (Ludwig et al., 2016). Readings of chip hybridization signals had been normalized according to Huber et al. (2002).

XhoI and NotI sites of psiCheck-2 (Promega). EcoRV digestion and DNA sequencing were used to confirm the identity of the recombinant clones. A couple of oligonucleotides representing the inverted target sequences were cloned into the psiCheck-2 vector by similar approach, in order to obtain the control plasmids (indicated as I).

\section{Cell Cultures, Transfections, and Luciferase Assay}

The human lung cell line A549 was cultured in DMEM containing $10 \%$ fetal bovine serum, $50 \mathrm{U} / \mathrm{ml}$ penicillin, and $100 \mu \mathrm{g} / \mathrm{ml}$ streptomycin. The day before transfection, the cells were trypsinized and seeded in a medium without antibiotics in 12-well plates.

Transfections were performed with cells at $80-90 \%$ of confluence by using $3 \mu$ l of Lipofectamine 2000 (Invitrogen, Thermo Fisher Scientific) for $1 \mu \mathrm{g}$ of nucleic acids, as described by the manufacturer. A549 cells were transfected with $0.2 \mu \mathrm{g}$ of reporter constructs; miScript miRNA mimics and the control unrelated sequence AllStars Negative Control (Qiagen) were transfected at $50 \mathrm{nM}$. Spike-expressing plasmid pUNO1-SARS2$\mathrm{S}$ (InvivoGen) was transfected at $1 \mu \mathrm{g}$; after $6 \mathrm{~h}$, transfection mix was replaced with complete medium. The analyses were performed $48 \mathrm{~h}$ after transfection.

Luciferase assays were performed using the DualLuciferase Reporter Assay System (Promega) according to the manufacturer's protocol.

Experiments were independently repeated at least two times in triplicates.

Statistical comparisons were performed by Student's $t$-test, and a value of $p<0.05$ was considered significant.

\section{RNA Purification and Real-Time PCR Analysis}

Total RNA purification from cell cultures was performed by miRNeasy Mini Kit (Qiagen) according to the manufacturer's protocol. The RNA concentration was determined spectrophotometrically (NanoDrop 2000c, Thermo Fisher Scientific). RNA was retrotranscribed by SensiFAST cDNA Synthesis kit (Bioline). Then, standard SYBR Green Real-time qPCR assays were performed with the following primers: Spike, 5'-TCAACTCAGGACTTGTTCTTAC-3' and 5'-TGGT AGGACAGGTTATCAAAC-3' (Zhen and Berry, 2020); GAPDH (reference transcript), 5'-GAAGGTGAAGGTCGGAGTC-3' and $5^{\prime}$-GAAGATGGTGATGGGATTT-3'. The expression level of Spike was normalized to the reference gene by using the $2^{-\Delta \mathrm{Ct}}$ method.

\section{RESULTS}

\section{Prediction of miRNA Target Sites Within the SARS-CoV-2 Genome}

The SARS-CoV-2 genome is made of a positive strand RNA molecule of 29903 nucleotides, provided with a cap and poly(A) tail. It contains $10 \mathrm{ORF}$ encompassing most of the genomic sequence with very short spacing regions. ORF 1a and $1 \mathrm{~b}$ encode non-structural proteins, such as the RNAdependent RNA Polymerase and two proteases. The other ORFs encode structural proteins including $S$ protein and nucleocapsid polypeptide chains.

SARS-CoV-2 genomic segments potentially targeted by human microRNAs were searched by the computer programs 
RNAhybrid 2.2 and MirTarget which predicted 2654 and 857 pairings, respectively. Target sequences identified by both programs (about 600) were then filtered to select microRNAs expressed in the lung, the natural site of infection, and showing a perfect match of the seed region (nucleotides 2-8). Selection was based on the analysis of the first published SARS-CoV-2 genomic sequence belonging to the isolate Wuhan-Hu-1 (NC_045512.2) (Wu et al., 2020), but conservation of the target sites within the SARS-CoV-2 population was also considered. In particular, only those targets that were entirely conserved in at least $95 \%$ of the genomic sequences deposited at NCBI were selected. This procedure yielded eight highly conserved genomic sequences potentially targeted by lung microRNAs, six in ORFs $1 \mathrm{a}$ and $1 \mathrm{~b}$, one in ORF S, and one in ORF 10 (Figure 1). These data suggested that the identified miRNAs may be able to interact with the viral RNAs within infected cells, thus contributing to the regulation of SARS-CoV-2 gene expression.

\section{Experimental Validation}

The viral sequences potentially targeted by the miRNAs were subjected to a first validation test, based on luciferase reporter constructs transfected in a cell line. A549 cells were chosen as a cell model, since they derive from the lung, the natural site of SARS-CoV-2 infection, express ACE2 and TMPRSS2 protein required for the virus entry, and are also susceptible to the virus infection (Chu et al., 2020; Pierce et al., 2020). In brief, the putative miRNA target sequences were individually cloned downstream the Renilla reniformis luciferase ( $\mathrm{Rl}$ ) coding sequence into the psi-Check-2 vector; the reporter constructs were then transfected in A549 cells and the luciferase activity was measured $48 \mathrm{~h}$ after the transfection. The expected results were that the binding ability of an endogenously expressed lung miRNA to the viral target should be registered as a reduced luciferase activity, due to the repression of the reporter protein production in comparison to the control. In this validation screening, the control was represented by the parental vector that did not contain any viral sequence. For transfection normalization, the Renilla luciferase values were related to those of luciferase (Luc), whose coding sequence is also contained in the psiCheck-2 vector. In this experimental setting, five sequences out of the eight selected by computational analyses caused luciferase activity reduction, ranging from approximately $20 \%$ inhibition (miR-219a and miR-29a target sequences) to about 40\% (miR-15b target sequence) and 50\% (miR-30c and miR-378d target sequences) (Figure 2).

These target sequences were subjected to further controls to exclude the possibility of an inhibition due to other factors other than the direct binding of the miRNA to the viral sequences. Control reporter constructs were prepared, each containing an inverted target sequence (I). The silencing effect of each miRNA was then investigated by transfecting the reporter construct containing the viral target sequence (now denominated WT) and its inverted sequence (I) along with a specific miRNA mimic and their negative control molecule that did not match any target sequence. The I constructs allowed to register the maximum value of the uninhibited reporter activity; in comparison, all the WT reporter plasmids transfected with the control miRNA

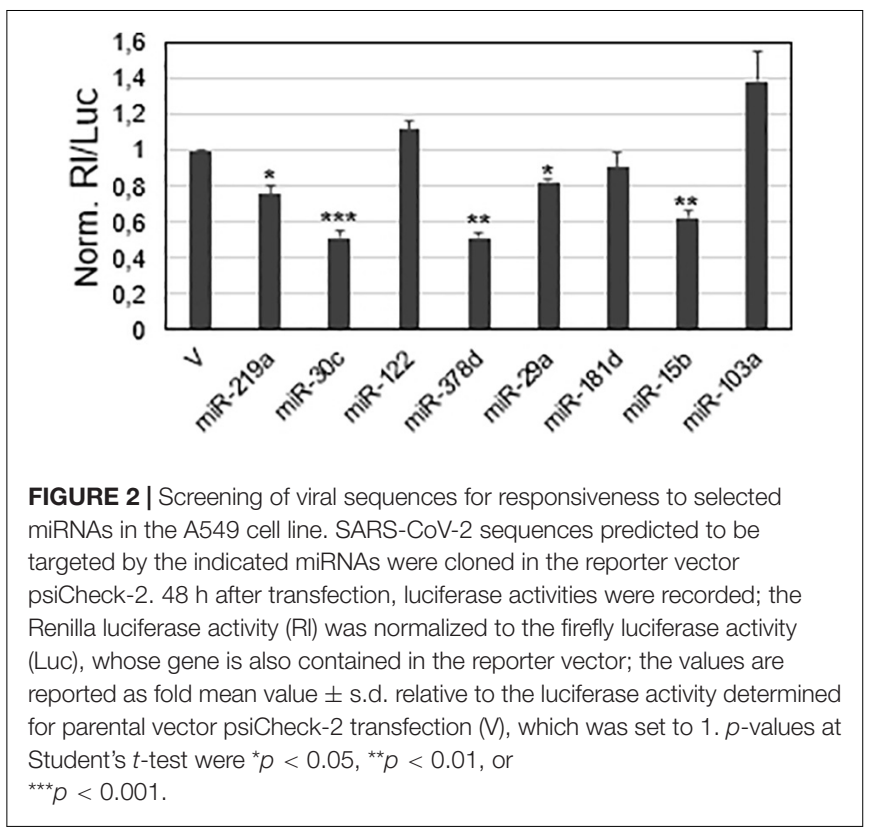

molecule showed a reduction of luciferase activity potentially due to endogenously expressed miRNAs, thus confirming the data from the previous screening. More importantly, their cotransfection along with the miRNA mimics showed an additional and significant reduction of luciferase activity, leading to a total inhibition ranging from approximately 60\% (miR-30c, miR$378 \mathrm{~d}$, and miR-15b) to more than $80 \%$ (miR-219a and miR-29a) in comparison to the controls (Figure 3A). Overall, the data demonstrate that the observed silencing effects can be attributed to the cellular miRNAs through a direct interaction with the viral sequences.

Given that miR-15b binds to the viral ORF encoding S protein, we further validated its binding to the $S$ transcript by exploiting the commercially available plasmid pUNO1-SARS2-S (pSpike) expressing the natural viral $S$ transcript from the Wuhan-Hu1 isolate. Co-transfection of pSpike along with miR-15b mimic showed a $72 \%$ reduction of Spike expression in comparison to co-transfection along with control unrelated miRNA mimic, demonstrating the ability of miR-15b to bind the target sequence in the context of the wild-type viral transcript (Figure 3B), with the resulting silencing effect.

\section{DISCUSSION}

In an evolutionary perspective, viruses and humans seem to be often engaged in a zero-sum game, whereby viruses infect host cells equipped with an array of mechanisms to evade the host defense responses and replicate, and in turn host cells build a defense mechanism to restrict the infection. Cellular or viral miRNAs play an emerging and important role in that biological arms race: viruses can produce their own miRNAs that can target viral transcripts to regulate their own viral cycle or target cellular transcripts to establish a favorable host environment; on 


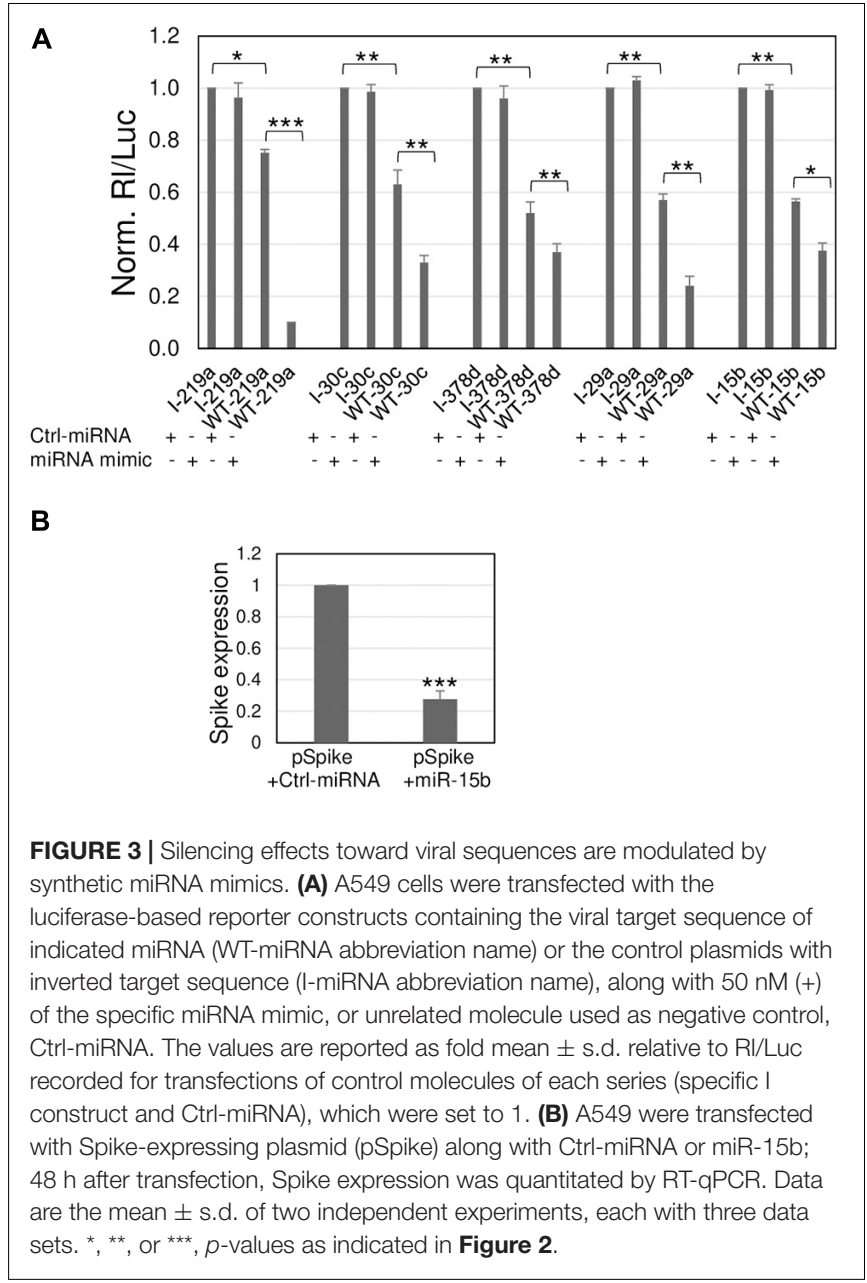

the other hand, host cells can counteract viral replication by breaking out an array of miRNAs that can directly target viral transcripts or indirectly affect virus replication by modulating host factors involved in the infection and pathogenesis (Cullen, 2006). Long-standing viral infections have probably generated a kind of equilibrium whereby viruses and humans coevolved and adapted each other also by exploiting miRNA machinery. One example is represented by HBV-human interaction: HBV infects the hepatocytes and express the transactivator $\mathrm{HBx}$ protein; as infection proceeds and viral load increases, the HBx level increases and leads to overexpression of miR-125a which, in turn, limits HBV replication (Mosca et al., 2014). This mechanism would balance the virus-host interaction providing a condition that is beneficial for both the host survival and the viral spread in humans; in fact, chronic hepatitis B often lasts decades. Thus, miRNA studies can contribute to understanding the mechanisms underlying the interplay between virus-host coexistence and provide a framework for the development of innovative therapeutic strategies.

Severe acute respiratory syndrome coronavirus 2 is a novel RNA virus for humans, still representing a lethal threat across the world. So far, few studies have predicted several cellular miRNAs targeting its transcripts, without experimental validation. Our research also started from a bioinformatic prediction by two different programs: RNAhybrid 2.2 and miRTarget. Then, criteria based on experimental evidence and virus preferential tropism were applied, besides the predicted binding energy and the perfect match of the seed region: confidence in the existence of the miRNA, based on number reads in miRBase ( $>20000$ ) and expression in the lung, the natural site of infection. Eight targets were found in viral ORFs $1 \mathrm{a}, 1 \mathrm{~b}$, and $S$ (Figure 1). Among them, miR-29a and miR-15b were also predicted to have several binding sites throughout the virus genome by Pierce et al. (2020); miR-29a emerged as a potential interactor with a viral genome also from in silico analysis of Jafarinejad-Farsangi et al. (2020). However, given the partial complementarity of miRNA/target pairing, the identification of the true targets is still challenging and requires experimental validation. Our experimental system consisted of luciferase-based reporter plasmids carrying viral target sequences or their inverted sequences singularly transfected or co-transfected with miRNA mimics in the lung A549 cell line. Transfection of the reporter plasmids resulted in a reduction of luciferase activity for five out of the eight potential binding sites, suggesting responsiveness to endogenously expressed miRNAs (Figure 2). Co-transfection of the reporter plasmids along with miRNA mimics led to a further and strong reduction of luciferase activity (up to $90 \%$ for miR219a), validating the interaction between the miRNAs and viral sequences (Figure 3A). Importantly, we also demonstrated that miR-15b was able to bind to the target sequence in the context of the native viral sequence encoding $S$ protein, strongly reducing its expression (Figure 3B).

It will be interesting to explore in specific cell contexts the cellular targets of the miRNAs identified by our analyses, in order to understand if they are also involved in the progression of SARS-CoV-2 infection and pathogenesis. So far, literature mainly reports their potential role in different types of tumors, the most studied field related to miRNA biology. In particular, miR219a has been involved in pituitary adenomas and gastric cancer (Wang Y. et al., 2020; Yang Z. et al., 2020); miR-30c in pancreatic ductal carcinoma, laryngeal cell carcinoma, osteosarcoma, gastric and lung cancer (Cao et al., 2017; Zhou et al., 2019; Li X. et al., 2020; Tanaka et al., 2020; Yang D. et al., 2020). Intriguingly, miR$30 \mathrm{c}$ has also been involved in the infection by Porcine epidemic diarrhea virus (PEDV), a member of the Alphacoronavirus family: the virus engages the SOCS1/miR-30c axis to escape IFNl-mediated antiviral immune responses (Wang C. et al., 2020). miR-29a-3p is involved in gastric and breast cancer, as well as colorectal cancer and hepatocarcinoma (Li Y. et al., 2020; Wang J. et al., 2020; Zheng et al., 2020; Pan et al., 2021; Qu et al., 2021); interestingly, miR-29a has been considered as a potential therapeutic target for HIV eradication, because of its binding to Nef viral protein, critical for viral persistence and release, and binding to the $3^{\prime}$ UTR region of the HIV genome, mediating the transport of the virus to P-bodies (Ahluwalia et al., 2008; Nathans et al., 2009). MiR-15b is probably one of the most extensively studied in relation to cancer pathways, since it shares most of its sequence, including the seed region, with the well-known miR-15a that together with the clustered 
miR-16 were the first miRNAs related to a tumor, the chronic lymphocytic leukemia (Calin et al., 2002); recently, both loci, miR-15a/16-1 and miR-15b/16-2, have been shown to drive the pathogenesis of acute myeloid leukemia (Lovat et al., 2020). miR$15 \mathrm{~b}$ has also been related to lung cancer (Wang et al., 2017; Guo et al., 2021). More importantly, two independent studies linked miR-15b to SARS-CoV-2. In the study of Kim et al. (2020), miR-15b has been predicted to bind to the genome sequences of severe acute respiratory syndrome coronavirus (SARS-CoV), Middle East respiratory-related coronavirus (MERS-CoV), and SARS-CoV-2; in addition, miR-15b was found downregulated in hamster infected lung compared to the lung samples before the infection. Consistently, Tang et al. (2020) found deregulated miR-15b expression also in red blood-depleted whole blood samples from moderate and severe COVID-19 patients and healthy donors; prediction of the miR-15b targetome and related biological pathways also supported its contribution to the disease pathogenesis, along with miR-146a-5p, miR-21-5p, and miR-142$3 \mathrm{p}$, all indicated as potential biomarkers of severe COVID-19 and as candidate therapeutic targets. Besides the study of Kim et al. (2020) on miR-15b-5p, other recent computational studies take into account the conservation of the miRNA targets in other human coronavirus (Chow and Salmena, 2020; Nersisyan et al., 2020). We then verified the presence in SARS-CoV and MERS$\mathrm{CoV}$ genomes of the other four experimentally validated targets and found that only the target of miR-29a-3p was present in the corresponding position SARS-CoV genome. This implies that for miR-219a-2-3p, miR-30c-5p, and miR-378d, the interactions are specific for SARS-CoV-2 whereas for miR-15b-5p and 29a-3p a wider binding ability to other coronavirus may be conceived.

Overall, those reports and our research indicate that further miRNA studies could greatly contribute to better understand the molecular mechanism underlying SARS-Co-V 2 infection and pathogenesis with relevant perspectives in diagnostics and therapeutics. Of note, our experimental data showed a strong downregulation of the reporter gene as a consequence of miRNA mimic binding to the viral sequences, up to $90 \%$ of inhibition in comparison to the control for a singularly transfected miRNA. In addition, the miR-15b mimic was able to inhibit the expression

\section{REFERENCES}

Ahluwalia, J. K., Khan, S. Z., Soni, K., Rawat, P., Gupta, A., Hariharan, M., et al. (2008). Human cellular microRNA hsa-miR-29a interferes with viral nef protein expression and HIV-1 replication. Retrovirology 5, 117-126.

Arisan, E. D., Dart, A., Grant, G. H., Arisan, S., Cuhadaroglu, S., Lange, S., et al. (2020). The prediction of miRNAs in SARS-CoV-2 genomes: hsa-miR databases identify 7 key miRs linked to host responses and virus pathogenicity-related KEGG pathways significant for comorbidities. Viruses 12:614.

Bhattacharyya, P., and Biswas, S. C. (2020). Small non-coding RNAs: do they encode answers for controlling SARS-CoV-2 in the future? Front. Microbiol. 11:571553. doi: 10.3389/fmicb.2020.571553

Calin, G. A., Dumitru, C. D., Shimizu, M., Bichi, R., Zupo, S., and Noch, E. (2002). Frequent deletions and down-regulation of micro- RNA genes miR15 and miR16 at 13q14 in chronic lymphocytic leukemia. Proc. Natl. Acad. Sci. U.S.A. 99, 15524-15529. doi: 10.1073/pnas.242606799

Cao, J. M., Li, G. Z., Han, M., Xu, H. L., and Huang, K. M. (2017). MiR-30c$5 \mathrm{p}$ suppresses migration, invasion and epithelial to mesenchymal transition of the natural Spike transcript when it is produced by a plasmid in A549 cells.

Furthermore, the viral target sequences selected by our analyses are fully conserved in more recent variants such as United Kingdom variant B.1.1.7 and South Africa 501Y.V2.

Further research is required to evaluate if the selected miRNA mimics are able to inhibit viral translation and replication; however, our studies represent a framework to envisage innovative therapeutic interventions based on treatments able to boost the cellular reservoir of antiviral microRNAs, such as formulations of individual synthetic miRNA mimics or their cocktails as a frontline treatment for COVID-19 patients or as a preventive strategy to heighten the protective capacity of cells against the infection. Related to the last point, it would be also interesting to quantify the expression of the identified miRNAs in large cohorts of patients with different severity of the disease to understand if the potential antiviral miRNAs could have a role in the individual susceptibility to SARS-Co-V-2 infection, viral insults, and patient outcome.

\section{DATA AVAILABILITY STATEMENT}

The original contributions presented in the study are included in the article, further inquiries can be directed to the corresponding authors.

\section{AUTHOR CONTRIBUTIONS}

$\mathrm{CS}$ and AD conducted all the experiments. AR and NP conceived the study, interpreted the results, and wrote the manuscript. All authors approved the submitted version.

\section{FUNDING}

AR and NP were supported by the VALERE grants from University of Campania "Luigi Vanvitelli".

of gastric cancer via targeting MTA1. Biomed. Pharmacother. 93, 554-560. doi: 10.1016/j.biopha.2017.06.084

Chen, Y., and Wang, X. (2020). miRDB: an online database for prediction of functional microRNA targets. Nucleic Acids Res. 48, D127-D131. doi: 10.1093/ nar/gkz757

Chow, J. T., and Salmena, L. (2020). Prediction and analysis of SARS-CoV-2 targeting MicroRNA in human lung epithelium. Genes 11:1002. doi: 10.3390/ genes11091002

Chu, H., Chan, J. F., Yuen, T. T., Shuai, H., Yuan, S., Wang, Y., et al. (2020). Comparative tropism, replication kinetics, and cell damage profiling of SARS-CoV-2 and SARS-CoV with implications for clinical manifestations, transmissibility, and laboratory studies of COVID-19: an observational study. Lancet Microbe 1, e14-e23. doi: 10.1016/S2666-5247(20)30004-5

Cullen, B. R. (2006). Viruses and microRNAs. Nat. Genet. 38, S25-S30. doi: 10. $1038 /$ ng1793

Demirci, M. D. S., and Adan, A. J. P. (2020). Computational analysis of microRNAmediated interactions in SARS-CoV-2 infection. PeerJ 8:e9369. doi: 10.7717/ peerj.9369 
Guo, K., Qi, D., and Huang, B. (2021). LncRNA MEG8 promotes NSCLC progression by modulating the miR-15a-5p-miR-15b-5p/PSAT1 axis. Cancer Cell Int. 21:84. doi: 10.1186/s12935-021-01772-8

Guterres, A., Lima, C. H. D. A., Miranda, R. L., and Gadelha, M. R. (2020). What is the potential function of microRNAs as biomarkers and therapeutic targets in COVID-19? Infect. Genet. Evol. 85:104417.

Hoffmann, M., Kleine-Weber, H., Schroeder, S., Krüger, N., Herrler, T., Erichsen, S., et al. (2020). SARS-CoV-2 cell entry depends on ACE2 and TMPRSS2 and is blocked by a clinically proven protease inhibitor. Cell 181, 271-280. doi: 10.1016/j.cell.2020.02.052

Hosseini Rad Sm, A., and McLellan, A. D. (2020). Implications of SARS-CoV-2 mutations for genomic RNA structure and host microRNA targeting. Int. J. Mol. Sci. 21:4807.

Huang, J., Wang, F., Argyris, E., Chen, K., Liang, Z., Tian, H., et al. (2007). Cellular microRNAs contribute to HIV-1 latency in resting primary CD4+ T lymphocytes. Nat. Med. 13, 1241-1247. doi: 10.1038/nm1639

Huber, W., von Heydebreck, A., Sültmann, H., Poustka, A., and Vingron, M. (2002). Variance stabilization applied to microarray data calibration and to the quantification of differential expression. Bioinformatics 18(Suppl. 1), S96-S104. doi: 10.1093/bioinformatics/18.suppl_1.s96

Ivashchenko, A., Berillo, O., Pyrkova, A., and Niyazova, R. (2014). Binding sites of miR-1273 family on the mRNA of target genes. Biomed Res. Int. 2014:620530. doi: $10.1155 / 2014 / 620530$

Jafarinejad-Farsangi, S., Jazi, M. M., Rostamzadeh, F., and Hadizadeh, M. (2020). High affinity of host human microRNAs to SARS-CoV-2 genome: an in silico analysis. Noncoding RNA Res. 5, 222-231. doi: 10.1016/j.ncrna.2020.11.005

Kim, W. R., Park, E. G., Kang, K., Lee, S., Kim, B., and Kim, H. (2020). Expression analyses of microRNAs in hamster lung tissues infected by SARS-CoV-2. Mol. Cells 43, 953-963. doi: 10.14348/molcells.2020.0177

Krüger, J., and Rehmsmeier, M. (2006). RNAhybrid: microRNA target prediction easy, fast and flexible. Nucleic Acids Res. 34, W451-W454. doi: 10.1093/nar/ gkl243

Li, H., Jiang, J. D., and Peng, Z. G. (2016). MicroRNA-mediated interactions between host and hepatitis C virus. World J. Gastroenterol. 22, 1487-1496. doi: 10.3748/wjg.v22.i4.1487

Li, X., Xu, F., Meng, Q., Gong, N., Teng, Z., Xu, R., et al. (2020). Long noncoding RNA DLEU2 predicts a poor prognosis and enhances malignant properties in laryngeal squamous cell carcinoma through the miR-30c-5p/PIK3CD/Akt axis. Cell Death Dis. 11:472. doi: 10.1038/s41419-020-2581-2

Li, Y., Zang, H., Zhang, X., and Huang, G. (2020). Exosomal circ-ZNF652 promotes cell proliferation, migration, invasion and glycolysis in hepatocellular carcinoma via miR-29a-3p/GUCD1 axis. Cancer Manage. Res. 12, 7739-7751. doi: 10.2147/CMAR.S259424

Lovat, F., Nigita, G., Distefano, R., Nakamura, T., Gasparini, P., Tomasello, L., et al. (2020). Combined loss of function of two different loci of miR-15/16 drives the pathogenesis of acute myeloid leukemia. Proc. Natl. Acad. Sci. U.S.A. 117, 12332-12340. doi: 10.1073/pnas.2003597117

Ludwig, N., Leidinger, P., Becker, K., Backes, C., Fehlmann, T., Pallasch, C., et al. (2016). Distribution of miRNA expression across human tissues. Nucleic Acids Res. 44, 3865-3877. doi: 10.1093/nar/gkw116

Mosca, N., Castiello, F., Coppola, N., Trotta, M. C., Sagnelli, C., Pisaturo, M., et al. (2014). Functional interplay between hepatitis B virus X protein and human miR-125a in HBV infection. Biochem. Biophys. Res. Commun. 449, 141-145. doi: 10.1016/j.bbrc.2014.05.009

Murakami, Y., Aly, H. H., Tajima, A., Inoue, I., and Shimotohno, K. (2009). Regulation of the hepatitis C virus genome replication by miR-199a. J. Hepatol. 50, 453-460. doi: 10.1016/j.jhep.2008.06.010

Nathans, R., Chu, C. Y., Serquina, A. K., Lu, C. C., Cao, H., and Rana, T. M. (2009). Cellular microRNA and P bodies modulate host-HIV-1 interactions. Mol. Cell 34, 696-709. doi: 10.1016/j.molcel.2009.06.003

Nersisyan, S., Engibaryan, N., Gorbonos, A., Kirdey, K., Makhonin, A., and Tonevitsky, A. (2020). Potential role of cellular miRNAs in coronavirus-host interplay. PeerJ 8:e9994. doi: 10.7717/peerj.9994

Nuovo, G. J., Wu, X., Volinia, S., Yan, F., di Leva, G., Chin, N., et al. (2010). Strong inverse correlation between microRNA-125b and human papillomavirus DNA in productive infection. Diagn. Mol. Pathol. 19, 135-143. doi: 10.1097/PDM. 0b013e3181c4daaa

Pan, H., Ding, Y., Jiang, Y., Wang, X., Rao, J., and Zhang, X. (2021). LncRNA LIFR-AS1 promotes proliferation and invasion of gastric cancer cell via
miR-29a-3p/COL1A2 axis. Cancer Cell Int. 21:7. doi: 10.1186/s12935-020-01 644-7

Pierce, J. B., Simion, V., Icli, B., Pérez-Cremades, D., Cheng, H. S., and Feinberg, M. W. (2020). Computational analysis of targeting SARS-CoV-2. viral entry proteins ACE2 and TMPRSS2, and interferon genes by host microRNAs. Genes 11:1354. doi: 10.3390/genes11111354

Potenza, N., Papa, U., Mosca, N., Zerbini, F., Nobile, V., and Russo, A. (2011). Human microRNA hsa-miR-125a-5p interferes with expression of hepatitis B virus surface antigen. Nucleic Acids Res. 39, 5157-5163. doi: 10.1093/nar/ gkr067

Potenza, N., Castiello, F., Panella, M., Colonna, G., Ciliberto, G., Russo, A., et al. (2016). Human miR-544a modulates SELK expression in hepatocarcinoma cell lines. PLoS One 11:e0156908. doi: 10.1371/journal.pone. 0156908

Qu, F., Zhu, B., Hu, Y., Mao, Q., and Feng, Y. (2021). LncRNA HOXA-AS3 promotes gastric cancer progression by regulating miR-29a-3p/LT $\beta R$ and activating NF-кB signaling. Cancer Cell Int. 21:118. doi: 10.1186/s12935-02101827-w

Rehmsmeier, M., Steffen, P., Hochsmann, M., and Giegerich, R. (2004). Fast and effective prediction of microRNA/target duplexes. RNA 10, 1507-1517. doi: 10.1261/rna.5248604

Russo, A., and Potenza, N. (2011). Antiviral effects of human microRNAs and conservation of their target sites. FEBS Lett. 585, 2551-2555. doi: 10.1016/j. febslet.2011.07.015

Rzeszutek, I., and Singh, A. (2020). Small RNAs, Big Diseases. Int. J. Mol. Sci. 21:5699.

Sagnelli, E., Potenza, N., Onorato, L., Sagnelli, C., Coppola, N., and Russo, A. (2018). Micro-RNAs in hepatitis B virus-related chronic liver diseases and hepatocellular carcinoma. World J Hepatol. 10, 558-570. doi: 10.4254/wjh.v10. i9.558

Sungnak, W., Huang, N., Bécavin, C., Berg, M., Queen, R., Litvinukova, M., et al. (2020). SARS-CoV-2 entry factors are highly expressed in nasal epithelial cells together with innate immune genes. Nat. Med. 26, 681-687. doi: 10.1038/ s41591-020-0868-6

Tanaka, T., Okada, R., Hozaka, Y., Wada, M., Moriya, S., Satake, S., et al. (2020). Molecular pathogenesis of pancreatic ductal adenocarcinoma: impact of miR$30 c-5 p$ and miR-30c-2-3p regulation on oncogenic genes. Cancers 12:2731. doi: $10.3390 /$ cancers 12102731

Tang, H., Gao, Y., Li, Z., Miao, Y., Huang, Z., and Liu, X. (2020). The noncoding and coding transcriptional landscape of the peripheral immune response in patients with COVID-19. Clin. Transl. Med. 10:e200. doi: 10.1002/ctm2. 200

Trobaugh, D. W., and Klimstra, W. B. (2017). MicroRNA regulation of RNA virus replication and pathogenesis. Trends Mol. Med. 23, 80-93. doi: 10.1016/j. molmed.2016.11.003

Umbach, J. L., and Cullen, B. R. (2009). The role of RNAi and microRNAs in animal virus replication and antiviral immunity. Genes Dev. 23, 1151-1164. doi: 10.1101/gad.1793309

Wang, C., Shan, L., Qu, S., Xue, M., Wang, K., Fu, F., et al. (2020). The coronavirus PEDV evades Type III interferon response through the miR-30c-5p/SOCS1 axis. Front. Microbiol. 11:1180. doi: 10.3389/fmicb.2020.01180

Wang, H., Zhan, Y., Jin, J., Zhang, C., and Li, W. (2017). MicroRNA-15b promotes proliferation and invasion of non-small cell lung carcinoma cells by directly targeting TIMP2. Oncol. Rep. 37, 3305-3312. doi: 10.3892/or.2017.5604

Wang, J., Huang, K., Shi, L., Zhang, Q., and Zhang, S. (2020). CircPVT1 promoted the progression of breast cancer by regulating MiR-29a-3p-mediated AGR2HIF- $1 \alpha$ pathway. Cancer Manag. Res. 12, 11477-11490. doi: 10.2147/CMAR. S265579

Wang, Y., Zhao, J., Zhang, C., Wang, P., Huang, C., and Peng, H. (2020). MiR219a-2-3p suppresses cell proliferation and promotes apoptosis by targeting MDM2/p53 in pituitary adenomas cells Biosci. Biotechnol. Biochem. 84, $911-$ 918. doi: 10.1080/09168451.2020.1715780

Wu, F., Zhao, S., Yu, B., Chen, Y. M., Wang, W., Song, Z. G., et al. (2020). A new coronavirus associated with human respiratory disease in China. Nature 579, 265-269. doi: 10.1038/s41586-020-2008-3

Yang, D., Liu, K., Fan, L., Liang, W., Xu, T., Jiang, W., et al. (2020). LncRNA RP11-361F15.2 promotes osteosarcoma tumorigenesis by inhibiting M2-Like polarization of tumor-associated macrophages of CPEB4. Cancer Lett. 31, 33-49. doi: 10.1016/j.canlet.2019.12.041 
Yang, Z., Dong, X., Pu, M., Yang, H., Chang, W., Ji, F., et al. (2020). LBX2-AS1/miR219a-2-3p/FUS/LBX2 positive feedback loop contributes to the proliferation of gastric cancer. Gastric Cancer 23, 449-463. doi: 10.1007/s10120-019-01019-6

Zhang, G. L., Li, Y. X., Zheng, S. Q., Liu, M., Li, X., and Tang, H. (2010). Suppression of hepatitis B virus replication by microRNA-199a-3p and microRNA-210. Antiviral Res. 88, 169-175. doi: 10.1016/j.antiviral.2010.08.008

Zhen, W., and Berry, G. J. (2020). Development of a new multiplex real-time RT-PCR assay for Severe Acute Respiratory Syndrome Coronavirus 2 (SARSCoV-2) detection. J. Mol. Diagn. 22, 1367-1372. doi: 10.1016/j.jmoldx.2020.09. 004

Zheng, X. Y., Cao, M. Z., Ba, Y., Li, Y. F., and Ye, J. L. (2020). LncRNA testisspecific transcript, Y-linked 15 (TTTY15) promotes proliferation, migration and invasion of colorectal cancer cells via regulating miR-29a-3p/DVL3 axis. Cancer Biomark. (pre-press), 1-11. doi: 10.3233/CBM-201709

Zhou, Y., Shi, H., Du, Y., Zhao, G., Wang, X., Li, Q., et al. (2019). IncRNA DLEU2 modulates cell proliferation and invasion of non-small cell lung cancer by regulating miR-30c-5p/SOX9 axis. Aging 11, 7386-7401. doi: 10.18632/aging. 102226
Zhu, N., Zhang, D., Wang, W., Li, X., Yang, B., Song, J., et al. (2020). A novel coronavirus from patients with pneumonia in China, 2019. N. Engl. J. Med. 382, 727-733. doi: 10.1056/NEJMoa2001017

Ziegler, C. G. K., Allon, S. J., Nyquist, S. K., Mbano, I. M., Miao, V. N., and Tzouanas, C. N. (2020). SARS-CoV-2 receptor ACE2 is an interferon-stimulated gene in human airway epithelial cells and is detected in specific cell subsets across tissues. Cell 181, 1016-1035.e19.

Conflict of Interest: The authors declare that the research was conducted in the absence of any commercial or financial relationships that could be construed as a potential conflict of interest.

Copyright (C) 2021 Siniscalchi, Di Palo, Russo and Potenza. This is an open-access article distributed under the terms of the Creative Commons Attribution License (CC BY). The use, distribution or reproduction in other forums is permitted, provided the original author(s) and the copyright owner(s) are credited and that the original publication in this journal is cited, in accordance with accepted academic practice. No use, distribution or reproduction is permitted which does not comply with these terms. 